\title{
Asymptotic Analysis of Three Dimensional Navier-Stokes Equations for Compressible Nonlinearly Viscous Fluids
}

\author{
Rostislav Vodák \\ Communicated by Tudor Ratiu, received September 2, 2008.
}

\begin{abstract}
In this paper, an asymptotic two dimensional model for compressible nonlinearly viscous fluids is rigorously derived as a limit of corresponding three dimensional models using asymptotic analysis with respect to the thickness of the respective three dimensional domains.
\end{abstract}

\section{Contents}

1. Introduction 299

2. Preliminaries 300

3. Proof of theorem $1 \quad 304$

References $\quad 310$

\section{Introduction}

A rigorous derivation of low-dimensional models from high-dimensional ones is an important area of asymptotic analysis. The first attempts were done in linear elasticity (see for instance [2], [3] and references therein for shells and plates, and $[\mathbf{1}],[\mathbf{7}],[\mathbf{8}],[\mathbf{1 7}]$ for beams and rods). The technique of the asymptotic analysis was originally based on an asymptotic expansion but the same results can be derived by a suitable scaling. These approaches can be applied to incompressible fluids, as it was presented in [6], [14] and [19]. In case of barotropic compressible fluids, this technique fails because of high oscillations in the pressure terms (see [18]). The alternative theory developed in $[\mathbf{1 2}]$ and $[\mathbf{1 3}]$ is based on function spaces depending on a sequence of thin domains.

In this paper, a suitable scaling is applied to a three dimensional model for compressible nonlinearly viscous fluids in thin cylinders, where nonlinearities appear in the stress tensor but not in the pressure term. We rigorously derive a two

1991 Mathematics Subject Classification. 35, 76.

Key words and phrases. Navier-Stokes equations, Asymptotic analysis, viscous fluids. 
dimensional model as the limit of a sequence of three dimensional models in thin cylinders. Moreover, for slip boundary conditions, this limit model coincides with the two dimensional Navier-Stokes equations for compressible nonlinearly viscous fluids. An important ingredient of the proof is the approximation of the first two components of the velocity fields by their mean values in the variable, which corresponds to the small proportion. We also discuss the form of Navier's boundary conditions which can affect the limit equations and which involve slip boundary conditions.

This paper is organized as follows: In Section 2, we introduce the basic notation used throughout the paper and state the main result. Section 3 contains the proof of the main result.

\section{Preliminaries}

In this paper, the motion of a compressible fluid, which is determined by the instantaneous values of the density $\bar{\rho}_{\epsilon}$ and the velocity $\overline{\mathbf{u}}_{\epsilon}=\left(\bar{u}_{1, \epsilon}, \bar{u}_{2, \epsilon}, \bar{u}_{3, \epsilon}\right)$, is studied in thin domains. If the motion is smooth, the time evolution of these quantities is governed by

\section{- Continuity Equation}

$$
\partial_{t} \bar{\rho}_{\epsilon}+\operatorname{div}\left(\bar{\rho}_{\epsilon} \overline{\mathbf{u}}_{\epsilon}\right)=0
$$

- Momentum Equation

$$
\partial_{t}\left(\bar{\rho}_{\epsilon} \overline{\mathbf{u}}_{\epsilon}\right)+\operatorname{div}\left(\bar{\rho}_{\epsilon} \overline{\mathbf{u}}_{\epsilon} \otimes \overline{\mathbf{u}}_{\epsilon}\right)+\bar{\nabla} \bar{p}_{\epsilon}=\operatorname{div} \overline{\mathbb{S}}_{\epsilon}+\bar{\rho}_{\epsilon} \overline{\mathbf{f}}_{\epsilon}
$$

in $\Omega_{\epsilon}$, where $\bar{p}_{\epsilon}$ is the pressure, $\overline{\mathbb{S}}_{\epsilon}$ is the viscous stress tensor and $\overline{\mathbf{f}}_{\epsilon}$ stands for external forces. To define $\Omega_{\epsilon}$ we assume a referential domain $\Omega:=S \times(0,1)$, $S \subset \mathbb{R}^{2}$ and $\partial S \in C^{0,1}$. Using the mapping

$$
\bar{x}=\mathbf{R}_{\epsilon}(x)=\left(x_{1}, x_{2}, \epsilon x_{3}\right), x=\left(x_{1}, x_{2}, x_{3}\right) \in \Omega,
$$

we can define $\Omega_{\epsilon}:=S \times(0, \epsilon)$. $\mathbf{n}_{\epsilon}$ and $\mathbf{n}$ denote unit outward normals to $\partial \Omega_{\epsilon}$ and $\partial \Omega$, respectively. We denote by "." scalar product, " $\times$ " Cartesian product and ":" scalar product of tensors. Without danger of confusion, we adopt $|\cdot|$ for the Euclidean norm in $\mathbb{R}^{3}$ or $\mathbb{R}^{9}$ generated by "." or ":", respectively, absolute value and the Lebesgue measure of some measurable set. By the symbol "-" and index $\epsilon$ we emphasize the fact that the domain is $\Omega_{\epsilon}$.

In this paper we pay attention to an isothermal gas, which means

$$
\bar{p}_{\epsilon}\left(\bar{\rho}_{\epsilon}\right)=c \bar{\rho}_{\epsilon},
$$

where we put $c=1$. We also restrict ourselves only to non-Newtonian fluids, i.e.

$$
\overline{\mathbb{S}}_{\epsilon}=P\left(\left|\bar{D} \overline{\mathbf{u}}_{\epsilon}\right|\right) \bar{D} \overline{\mathbf{u}}_{\epsilon},
$$

where $\bar{D}$ is the symmetric part of the gradient and the function $P$ will be specified later. We complete (2.1)-(2.5) by the set of the Navier boundary conditions

$$
\begin{gathered}
\mathbf{t}_{\epsilon} \cdot\left(P\left(\left|\bar{D} \overline{\mathbf{u}}_{\epsilon}\right|\right) \bar{D} \overline{\mathbf{u}}_{\epsilon} \mathbf{n}_{\epsilon}\right)+q \overline{\mathbf{u}}_{\epsilon} \cdot \mathbf{t}_{\epsilon}=0 \text { on } \partial S \times(0, \epsilon) \times(0, T), \\
\mathbf{t}_{\epsilon} \cdot\left(P\left(\left|\bar{D} \overline{\mathbf{u}}_{\epsilon}\right|\right) \bar{D} \overline{\mathbf{u}}_{\epsilon} \mathbf{n}_{\epsilon}\right)+h(\epsilon) \overline{\mathbf{u}}_{\epsilon} \cdot \mathbf{t}_{\epsilon}=0 \text { on }[(S \times\{0\}) \cup(S \times\{\epsilon\})] \times(0, T), \\
\overline{\mathbf{u}}_{\epsilon} \cdot \mathbf{n}_{\epsilon}=0 \text { on } \partial \Omega_{\epsilon} \times(0, T)
\end{gathered}
$$

to ensure well-posedness of the problem. Here, $\mathbf{t}_{\epsilon}$ is any vector from the corresponding tangent plane, $q$ is a nonnegative constant and $h(\epsilon) \sim o(\epsilon)$ or $h(\epsilon) \sim O(\epsilon)$. The choice of $h_{\epsilon}$ will be discussed during derivation of weak convergences of velocity 
fields and their consequences. Finally, we prescribed the initial conditions for the density and momentum:

$$
\bar{\rho}_{\epsilon}(x, 0)=\bar{\rho}_{0, \epsilon}(x) \geq 0,\left(\bar{\rho}_{\epsilon} \overline{\mathbf{u}}_{\epsilon}\right)(x, 0)=\left(\bar{\rho}_{\epsilon} \overline{\mathbf{u}}_{\epsilon}\right)_{0}(x) \text { in } \Omega_{\epsilon} .
$$

We further adopt the standard notation $L^{p}(Q), W^{1, p}(Q)$ and $L^{p}(0, T ; X), p \in$ $[1, \infty]$, for Lebesgue, Sobolev and Bochner spaces, respectively. The corresponding norms read $\|\cdot\|_{p, Q},\|\cdot\|_{1, p, Q}$ and $\|\cdot\|_{L^{p}(0, T ; X)}$. Spaces of continuous and continuously differentiable functions up to order $m$ are denoted by $C^{m}(\bar{Q})$ with the norm $\|$. $\|_{C^{m}(\bar{Q})}$ and by $C^{m}([0, T] ; X)$ with the norm $\|\cdot\|_{C^{m}([0, T] ; X)}$. By $\mathcal{D}(Q)$ we denote the space of smooth, compactly supported functions equipped with the topology of locally uniform convergence.

We now pay attention to the definition of Orlicz spaces because they are not so widespread. By $L_{\Phi}(Q)$ we denote the Orlicz space of such functions that

$$
\|v\|_{\Phi, Q}:=\sup _{w \in A} \int_{Q} v w d y<+\infty, A=\left\{w ; \int_{Q} \Psi(|w|) d y<1\right\},
$$

where $\Psi$ is a complementary function to the Young function $\Phi$ and corresponds to conjugate exponents in the theory of Lebesgue spaces. Let us point out that in the theory of Orlicz spaces the relations hold:

$$
E_{\Phi}(Q):={\overline{C_{0}^{\infty}(Q)}}^{\|\cdot\|_{\Phi, Q}} \subseteq \widetilde{L}_{\Phi}(Q) \subseteq L_{\Phi}(Q)
$$

for $\widetilde{L}_{\Phi}(Q):=\left\{v ; \int_{Q} \Phi(|v|) d y<+\infty\right\}$, where the above three sets do not generally coincide in contrast to Lebesgue spaces. To ensure the coincidence of the above sets and spaces, the Young function $\Phi$ must satisfy the $\Delta_{2}$-condition:

$$
\Phi(2 z) \leq c \Phi(z), z \geq z_{0} \geq 0 .
$$

The Sobolev Orlicz spaces $W^{1} L_{\Phi}(Q)$ involve functions with this finite norm

$$
\|v\|_{1, \Phi, Q}:=\sqrt{\sum_{\alpha,|\alpha| \leq 1}\left\|D^{\alpha} v\right\|_{\Phi, Q}^{2}}<+\infty .
$$

The dual spaces to the spaces $W_{0}^{1, p}(Q)$ and $W_{0}^{1} L_{\Phi}(Q)$ are denoted by $W^{-1, p^{\prime}}(Q)$ and $W^{-1} L_{\Psi}(Q)$, respectively.

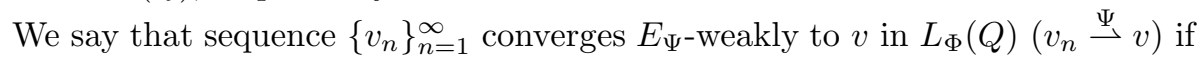

$$
\int_{Q} v_{n} w d y \rightarrow \int_{Q} v w d y \text { for any } w \in E_{\Psi}(Q) .
$$

In addition, any Orlicz space $L_{\Phi}(Q)$ is $E_{\Psi \text {-weakly compact. }}$

To derive suitable estimates we use the Young inequality

$$
|u v| \leq \Phi(|u|)+\Psi(|v|)
$$

and Hölder inequality

$$
\int_{Q}|u v| d y \leq\|u\|_{\Phi, Q}\|v\|_{\Psi, Q}
$$

which hold for a pair of complementary functions $\Phi$ and $\Psi$. For a detailed introduction to the theory of Orlicz spaces we refer to $[\mathbf{9}]$.

In this paper we will use the notation $\Phi_{1}(z)$ and $\Phi_{\gamma}(z), \gamma>1$, and $M(z)$ for the Young functions $z \ln (1+z),(1+z) \ln ^{\gamma}(1+z)$ and $e^{z}-z-1$ for $z \geq 0$, respectively. $\Psi_{1}(z), \Psi_{\gamma}(z)$ and $\widetilde{M}(z)$ then denote their complementary functions. 
The variational formulation of $(2.1)-(2.7)$ is

$$
\begin{gathered}
\int_{0}^{T} \int_{\Omega_{\epsilon}}\left[\bar{\rho}_{\epsilon} \partial_{t} \bar{\varphi}+\bar{\rho}_{\epsilon} \overline{\mathbf{u}}_{\epsilon} \cdot \bar{\nabla} \bar{\varphi}\right] d \bar{x} d t=0, \forall \bar{\varphi} \in \mathcal{D}\left(\mathbb{R}^{3} \times(0, T)\right) \\
\int_{0}^{T} \int_{\Omega_{\epsilon}}\left[\bar{\rho}_{\epsilon} \overline{\mathbf{u}}_{\epsilon} \cdot \partial_{t} \bar{\psi}+\bar{\rho}_{\epsilon} \overline{\mathbf{u}}_{\epsilon} \otimes \overline{\mathbf{u}}_{\epsilon}: \bar{D} \bar{\psi}+\bar{\rho}_{\epsilon} \operatorname{div} \bar{\psi}\right] d \bar{x} d t= \\
\quad \int_{0}^{T} \int_{\Omega_{\epsilon}}\left[P\left(\left|\bar{D} \overline{\mathbf{u}}_{\epsilon}\right|\right) \bar{D} \overline{\mathbf{u}}_{\epsilon}: \bar{D} \bar{\psi}-\bar{\rho}_{\epsilon} \overline{\mathbf{f}}_{\epsilon} \cdot \bar{\psi}\right] d \bar{x} d t+ \\
q \int_{0}^{T} \int_{\partial S \times(0, \epsilon)} \overline{\mathbf{u}}_{\epsilon} \cdot \bar{\psi} d S_{\epsilon} d t+h(\epsilon) \int_{0}^{T} \int_{(S \times\{0\}) \cup(S \times\{\epsilon\})} \overline{\mathbf{u}}_{\epsilon} \cdot \bar{\psi} d S_{\epsilon} d t
\end{gathered}
$$

for any $\bar{\psi} \in C_{0}^{\infty}\left(0, T ; C^{\infty}\left(\bar{\Omega}_{\epsilon}\right)^{3}\right),\left.\bar{\psi} \cdot \mathbf{n}_{\epsilon}\right|_{\partial \Omega_{\epsilon} \times(0, T)}=0$.

Assume the function $P$ satisfies

(1)

$$
\int_{\Omega_{\epsilon}} P(|\bar{D} \overline{\mathbf{v}}|)|\bar{D} \overline{\mathbf{v}}|^{2} d \bar{x} \geq \int_{\Omega_{\epsilon}} M(|\bar{D} \overline{\mathbf{v}}|) d \bar{x}
$$

$$
\int_{\Omega_{\epsilon}}(P(|\bar{D} \overline{\mathbf{v}}|) \bar{D} \overline{\mathbf{v}}-P(|\bar{D} \overline{\mathbf{w}}|) \bar{D} \overline{\mathbf{w}}):(\bar{D} \overline{\mathbf{v}}-\bar{D} \overline{\mathbf{w}}) d \bar{x} \geq 0
$$

$$
P(z)|z|^{2} \text { is a convex function for } z \geq 0
$$

$$
\int_{\Omega_{\epsilon}} \widetilde{M}(P(|\bar{D} \overline{\mathbf{v}}|)|\bar{D} \overline{\mathbf{v}}|) d \bar{x} \leq c\left(1+\int_{\Omega_{\epsilon}} M(|\bar{D} \overline{\mathbf{v}}|) d \bar{x}\right)
$$

$P(|\bar{D} \overline{\mathbf{v}}-\lambda \bar{D} \overline{\mathbf{w}}|)(\bar{D} \overline{\mathbf{v}}-\lambda \bar{D} \overline{\mathbf{w}}) \stackrel{M}{\rightarrow} P(|\bar{D} \overline{\mathbf{v}}|) \bar{D} \overline{\mathbf{v}}$

for $\lambda \rightarrow 0$.

The equations for compressible nonlinearly viscous fluids with non-slip boundary conditions have at least one weak solution under assumptions $(2.14)-(2.18)$ in a sufficiently regular domain (see $[\mathbf{1 0}]$ and $[\mathbf{1 1}]$ ). The case of slip boundary conditions can be treated similarly as the barotropic case (see [15]). To relax the assumptions on the regularity of the boundary of the domain $\Omega_{\epsilon}$, we can use the approach presented in [5]. Here, we use the Navier boundary conditions (2.6), because slip boundary conditions are their special cases $(h(\epsilon)=q=0)$. In case of non-slip boundary conditions, the limit of velocity fields is equal to zero and thus we omit it.

We now give a brief comment to the usage of Orlicz spaces. The first reason is that for the Young function $M$ with so rapid growth the results are independent of dimension. The second one is technical and it is hidden in (2.4). To obtain some type of weak convergence, we need better integrability of $\bar{\rho}_{\epsilon}$. In contrast to the barotropic case, we can improve the integrability of density using the renormalized version of continuity equation (see (2.25) and (3.5)), where, in the worst case, we must be able to control the term $\bar{\rho}_{\epsilon} \operatorname{div} \overline{\mathbf{u}}_{\epsilon}$ with $\rho_{\epsilon}$ from the Orlicz spaces close to 
$L^{1}(\Omega)$. The same term must be treated during the derivation of the energy equality. But to derive the energy equality we have to use $\overline{\mathbf{u}}_{\epsilon}$ as a test function in (2.13) (using some suitable smoothing in time variable) and we have to control the convective term as well.

If we transform equations (2.12) and (2.13) to a referential domain $\Omega$ given by (2.3) and employ the scaling

$$
\begin{gathered}
u_{1, \epsilon}=\bar{u}_{1, \epsilon}, u_{2, \epsilon}=\bar{u}_{2, \epsilon}, u_{3, \epsilon}=\epsilon \bar{u}_{3, \epsilon}, \\
\psi_{1}=\bar{\psi}_{1, \epsilon}, \psi_{2}=\bar{\psi}_{2, \epsilon}, \psi_{3}=\epsilon \bar{\psi}_{3, \epsilon}, \\
f_{1}=\bar{f}_{1, \epsilon}, f_{2}=\bar{f}_{2, \epsilon}, f_{3}=\frac{\bar{f}_{3, \epsilon}}{\epsilon}
\end{gathered}
$$

where $\mathbf{f} \in L^{\infty}(\Omega \times(0, T))^{3}$, we arrive at the equations

$$
\int_{0}^{T} \int_{\Omega}\left[\rho_{\epsilon} \varphi_{t}+\rho_{\epsilon} \widetilde{\mathbf{u}}_{\epsilon} \cdot \nabla_{\epsilon} \varphi\right] d x d t=0
$$

for any $\varphi \in \mathcal{D}\left(\mathbb{R}^{3} \times(0, T)\right)$, where $\nabla_{\epsilon}=\left(\partial_{1}, \partial_{2}, \frac{\partial_{3}}{\epsilon}\right)$, and

$$
\begin{gathered}
\int_{0}^{T} \int_{\Omega}\left[\rho_{\epsilon} \widetilde{\mathbf{u}}_{\epsilon} \cdot \partial_{t} \widetilde{\psi}_{\epsilon}+\rho_{\epsilon} \widetilde{\mathbf{u}}_{\epsilon} \otimes \widetilde{\mathbf{u}}_{\epsilon}: D_{\epsilon} \widetilde{\psi}_{\epsilon}+\rho_{\epsilon} \operatorname{div}_{\epsilon} \widetilde{\psi}_{\epsilon}\right] d x d t= \\
\int_{0}^{T} \int_{\Omega}\left[P\left(\left|D_{\epsilon} \widetilde{\mathbf{u}}_{\epsilon}\right|\right) D_{\epsilon} \widetilde{\mathbf{u}}_{\epsilon}: D_{\epsilon} \widetilde{\psi}_{\epsilon}-\rho_{\epsilon} \mathbf{f} \cdot \psi\right] d x d t+ \\
q \int_{0}^{T} \int_{\partial S \times(0,1)} \widetilde{\mathbf{u}}_{\epsilon} \cdot \psi d S d t+\frac{h(\epsilon)}{\epsilon} \int_{0}^{T} \int_{(S \times\{0\}) \cup S \times\{1\})} \widetilde{\mathbf{u}}_{\epsilon} \cdot \psi d S d t
\end{gathered}
$$

for any $\psi=\left(\psi_{1}, \psi_{2}, \psi_{3}\right) \in C_{0}^{\infty}\left(0, T ; C^{\infty}(\bar{\Omega})^{3}\right),\left.\psi \cdot \mathbf{n}\right|_{\partial \Omega \times(0, T)}=0$, where $\widetilde{\mathbf{u}}_{\epsilon}=$ $\left(u_{1, \epsilon}, u_{2, \epsilon}, \frac{u_{3, \epsilon}}{\epsilon}\right), \mathbf{u}_{\epsilon}:=\left(u_{1, \epsilon}, u_{2, \epsilon}, u_{3, \epsilon}\right), \widetilde{\psi}_{\epsilon}=\left(\psi_{1}, \psi_{2}, \frac{\psi_{3}}{\epsilon}\right), \operatorname{div}_{\epsilon} \widetilde{\psi}_{\epsilon}:=\nabla_{\epsilon} \cdot \widetilde{\psi}_{\epsilon}$ and $D_{\epsilon} \phi$ is a symmetric tensor defined by

$$
D_{\epsilon} \phi:=\left(\begin{array}{lll}
\partial_{1} \phi_{1} & \frac{\partial_{2} \phi_{1}+\partial_{1} \phi_{2}}{2} & \frac{1}{2}\left(\frac{\partial_{3} \phi_{1}}{\epsilon^{\epsilon}}+\partial_{1} \phi_{3}\right) \\
\cdot & \partial_{2} \phi_{2} & \frac{1}{2}\left(\frac{\partial_{3} \phi_{2}}{\epsilon}+\partial_{2} \phi_{3}\right) \\
\cdot & \cdot & \frac{\partial_{3} \phi_{3}}{\epsilon}
\end{array}\right) .
$$

From the definition of $\Omega$ we immediately get $\left.\widetilde{\psi}_{\epsilon} \cdot \mathbf{n}\right|_{\partial \Omega \times(0, T)}=0$.

If we subject the corresponding renormalized continuity equations and energy equalities (see [4] and [11] for their original forms) to the same transformation we come to the relations

$$
\int_{0}^{T} \int_{\Omega} b\left(\rho_{\epsilon}\right) \partial_{t} \varphi+b\left(\rho_{\epsilon}\right) \widetilde{\mathbf{u}}_{\epsilon} \cdot \nabla_{\epsilon} \varphi+\left[\left(b\left(\rho_{\epsilon}\right)-\rho_{\epsilon} b^{\prime}\left(\rho_{\epsilon}\right)\right) \operatorname{div}_{\epsilon} \widetilde{\mathbf{u}}_{\epsilon}\right] \varphi d x d t=0
$$

for any $\varphi \in \mathcal{D}\left(\mathbb{R}^{3} \times(0, T)\right)$, and

$$
\begin{gathered}
\int_{\Omega}\left[\frac{\rho_{\epsilon}(t)\left|\widetilde{\mathbf{u}}_{\epsilon}(t)\right|^{2}}{2}+\Phi_{1}\left(\rho_{\epsilon}(t)\right)\right] d x+\int_{0}^{t} \int_{\Omega} P\left(\left|D_{\epsilon} \widetilde{\mathbf{u}}_{\epsilon}\right|\right)\left|D_{\epsilon} \widetilde{\mathbf{u}}_{\epsilon}\right|^{2} d x d s+ \\
q \int_{0}^{t} \int_{\partial S \times(0,1)}\left|\widetilde{\mathbf{u}}_{\epsilon}\right|^{2} d S d s+\frac{h(\epsilon)}{\epsilon} \int_{0}^{t} \int_{(S \times\{0\}) \cup S \times\{1\})}\left|\widetilde{\mathbf{u}}_{\epsilon}\right|^{2} d S d s= \\
\int_{0}^{t} \int_{\Omega} \rho_{\epsilon} \mathbf{f} \cdot \mathbf{u}_{\epsilon} d x d s+\int_{\Omega}\left[\frac{\left|\left(\rho_{\epsilon} \widetilde{\mathbf{u}}_{\epsilon}\right)_{0}\right|^{2}}{2 \rho_{0, \epsilon}}+\Phi_{1}\left(\rho_{0, \epsilon}\right)\right] d x
\end{gathered}
$$


for any $t \in[0, T]$. As the last step, we prescribe the following behavior of initial states:

$$
\begin{aligned}
& \int_{0}^{1} \frac{\left|\left(\rho_{\epsilon} \widetilde{\mathbf{u}}_{\epsilon}\right)_{0}\right|^{2}}{2 \rho_{0, \epsilon}} d x_{3} \rightarrow \frac{\left|(\rho \mathbf{u})_{0}\right|^{2}}{2 \rho_{0}} \\
& \int_{0}^{1} \Phi_{\gamma}\left(\rho_{0, \epsilon}\right) d x_{3} \rightarrow \Phi_{\gamma}\left(\rho_{0}\right) \text { in } L^{1}(S) \text { for } \epsilon \rightarrow 0
\end{aligned}
$$

where $\mathbf{u}=\left(u_{1}, u_{2}, 0\right)$ and $\gamma \geq 1$.

The main result is stated in the following theorem:

TheOREM 1. Take couples $\left(\rho_{\epsilon}, \mathbf{u}_{\epsilon}\right), \epsilon \in(0,1)$, such that $\rho_{\epsilon} \in L^{\infty}\left(0, T ; L_{\Phi_{\gamma}}(\Omega)\right)$, $\gamma \geq 2, \mathbf{u}_{\epsilon} \in L^{p}\left(0, T ; W^{1, p}(\Omega)^{3}\right)$ for any $p \geq 1,\left.\mathbf{u}_{\epsilon} \cdot \mathbf{n}\right|_{\partial \Omega \times(0, T)}=0$ and $D_{\epsilon} \widetilde{\mathbf{u}}_{\epsilon} \in$ $L_{M}(\Omega \times(0, T))^{9}$, which are weak solutions to the equations (2.22), (2.23) with the initial states $\rho_{0, \epsilon} \in L_{\Phi_{\gamma}}(\Omega), \gamma \geq 2, \frac{\left|\left(\rho_{\epsilon} \widetilde{\mathbf{u}}_{\epsilon}\right)_{0}\right|^{2}}{2 \rho_{0, \epsilon}} \in L^{1}(\Omega)$ satisfying $(2.27), h(\epsilon) \sim o(\epsilon)$ or $h(\epsilon) \sim O(\epsilon)$, and with the function $P$ satisfying (2.14)-(2.18). Assume that if $q=0$ then $S$ is not a circle. Then (passing to subsequences if necessary)

$$
\begin{gathered}
\rho_{\epsilon} \stackrel{*}{\rightarrow} \rho \text { in } L^{\infty}\left(0, T ; L_{\Phi_{\gamma}}(\Omega)\right), \rho_{\epsilon} \rightarrow \rho \text { in } C\left([0, T] ; W^{-1} L_{\Phi_{\gamma}}(\Omega)\right), \\
D_{\epsilon} \widetilde{\mathbf{u}}_{\epsilon} \stackrel{\bar{M}}{\rightarrow} D \mathbf{u}, \mathbf{u}_{\epsilon} \rightarrow \mathbf{u} \text { in } L^{p}\left(0, T ; W^{1, p}(\Omega)^{3}\right) \cap L^{2}\left(0, T ; L^{2}(\partial \Omega)^{3}\right),
\end{gathered}
$$

where $\rho=\rho\left(x_{1}, x_{2}\right), \mathbf{u}\left(x_{1}, x_{2}\right)=\left(u_{1}\left(x_{1}, x_{2}\right), u_{2}\left(x_{1}, x_{2}\right), 0\right),\left.\mathbf{u} \cdot \mathbf{n}\right|_{\partial S \times(0, T)}=0$ and the couple $(\rho, \mathbf{u})$ is a solution to (3.34) and (3.35) with $\hat{\rho}=\rho$, and satisfies the energy equality (3.36).

\section{Proof of theorem 1}

In this section, we derive such estimates and convergences which enable us to perform the limit in (2.22) and (2.23) and to derive the variational formulation of the two dimensional Navier-Stokes equations for isothermal nonlinearly viscous fluids. We then check the energy equality holds for a solution to the limit equations and we show how to treat nonlinearity in stress tensor. At the end we verify that density $\rho$ does not depend on the third variable.

First of all we use the $L^{p}$-version of the Korn inequality for $p>3$ to derive the estimate

$$
\begin{gathered}
\|\mathbf{v}\|_{1, p, \Omega}^{p} \leq c\left(\|D \mathbf{v}\|_{p, \Omega}^{p}+q\|\mathbf{v}\|_{p, \partial S \times(0,1)}^{p}\right) \leq \\
c\left(\|D \mathbf{v}\|_{p, \Omega}^{p}+c_{1}\|\mathbf{v}\|_{\infty, \partial S \times(0,1)}^{p}+c_{2}\left(c_{1}\right) q\|\mathbf{v}\|_{2, \partial S \times(0,1)}^{p}\right),
\end{gathered}
$$

where $c_{1}>0$ is sufficiently small. Using the imbedding $W^{1, p}(\Omega) \hookrightarrow C(\bar{\Omega})$ for $p>3$ we arrive at

$$
\|\mathbf{v}\|_{1, p, \Omega} \leq c\left(\|D \mathbf{v}\|_{p, \Omega}+q^{1 / p}\|\mathbf{v}\|_{2, \partial S \times(0,1)}\right) .
$$

Then the first integral on the right-hand side of (2.26) can be estimated as follows

$$
\begin{gathered}
\left|\int_{0}^{t} \int_{\Omega} \rho_{\epsilon} \mathbf{f} \cdot \mathbf{u}_{\epsilon} d x d s\right| \leq \int_{0}^{t}\left\|\mathbf{u}_{\epsilon}(s)\right\|_{\infty, \Omega}\|\mathbf{f}(s)\|_{\infty, \Omega} \int_{\Omega} \rho_{\epsilon}(s) d x d s \stackrel{(2.22)}{\leq} \\
c\left(\rho_{0, \epsilon}, \mathbf{f}\right) \int_{0}^{t}\left\|\mathbf{u}_{\epsilon}(s)\right\|_{1, p, \Omega} d s \stackrel{(2.10),(2.14),(2.24),(3.1)}{\leq}
\end{gathered}
$$




$$
c\left(\rho_{0, \epsilon}, \mathbf{f}\right)\left(c_{1} \int_{0}^{t} \int_{\Omega} P\left(\left|D_{\epsilon} \widetilde{\mathbf{u}}_{\epsilon}\right|\right)\left|D_{\epsilon} \widetilde{\mathbf{u}}_{\epsilon}\right|^{2} d x d s+c_{1} \int_{0}^{t} \int_{\partial S \times(0,1)}\left|\widetilde{\mathbf{u}}_{\epsilon}\right|^{2} d S d s+c_{2}\left(c_{1}\right)\right),
$$

where $c_{1}>0$ is sufficiently small. In view of (2.14), (2.26), (3.2) and the Gronwall lemma, we then obtain the boundedness of

$$
\begin{gathered}
\left\{\sqrt{\rho_{\epsilon}}\left|\widetilde{\mathbf{u}}_{\epsilon}\right|\right\}_{\epsilon \in(0,1)} \text { in } L^{\infty}\left(0, T ; L^{2}(\Omega)\right),\left\{\rho_{\epsilon}\right\}_{\epsilon \in(0,1)} \text { in } L^{\infty}\left(0, T ; L_{\Phi_{1}}(\Omega)\right), \\
\left\{D_{\epsilon} \widetilde{\mathbf{u}}_{\epsilon}\right\}_{\epsilon \in(0,1)} \text { in } L_{M}(\Omega \times(0, T))^{9},
\end{gathered}
$$

$$
\left\{\mathbf{u}_{\epsilon}\right\}_{\epsilon \in(0,1)} \text { in } L^{p}\left(0, T ; W^{1, p}(\Omega)^{3}\right) \cap L^{2}\left(0, T ; L^{2}(\partial \Omega)^{3}\right)
$$

for any $p \geq 1$. If we further put $\varphi=\varphi(t) \in C_{0}^{\infty}(0, T)$ as a test function in (2.25) with $b(z)=\Phi_{\gamma}(z), \gamma \geq 1$, we arrive at

$$
\int_{0}^{T} \int_{\Omega} \Phi_{\gamma}\left(\rho_{\epsilon}\right) \varphi^{\prime}(t)+\left[\left(\Phi_{\gamma}\left(\rho_{\epsilon}\right)-\rho_{\epsilon} \Phi_{\gamma}^{\prime}\left(\rho_{\epsilon}\right)\right) \operatorname{div}_{\epsilon} \widetilde{\mathbf{u}}_{\epsilon}\right] \varphi(t) d x d t=0 .
$$

Since $\Phi_{\gamma}(z)-z \Phi_{\gamma}^{\prime}(z)$ behaves asymptotically as $\Phi_{\gamma-1}(z)$ and $\Phi_{1}\left(\Phi_{\gamma-1}(z)\right) \leq c\left(\Phi_{\gamma}(z)+\right.$ 1) for $z \geq 0$, we deduce the estimate

$$
\begin{aligned}
& \left|\int_{0}^{T} \int_{\Omega}\left[\left(\Phi_{\gamma}\left(\rho_{\epsilon}\right)-\rho_{\epsilon} \Phi_{\gamma}^{\prime}\left(\rho_{\epsilon}\right)\right) \operatorname{div}_{\epsilon} \widetilde{\mathbf{u}}_{\epsilon}\right] \varphi(t) d x d t\right| \stackrel{(2.10),(2.14),(3.4)_{1}}{\leq} \\
& c(T)\left(\int_{0}^{T} \int_{\Omega} \Phi_{\gamma}\left(\rho_{\epsilon}\right) d x d t+\int_{0}^{T} \int_{\Omega} P\left(\left|D_{\epsilon} \widetilde{\mathbf{u}}_{\epsilon}\right|\right)\left|D_{\epsilon} \widetilde{\mathbf{u}}_{\epsilon}\right|^{2} d x d t+1\right) .
\end{aligned}
$$

As a consequence of (2.27), (3.5), (3.6) and the Gronwall lemma we obtain the boundedness of

$$
\left\{\rho_{\epsilon}\right\}_{\epsilon \in(0,1)} \text { in } L^{\infty}\left(0, T ; \widetilde{L}_{\Phi_{\gamma}}(\Omega)\right), \gamma \geq 1 .
$$

In addition, it follows from (2.22), (3.4) and (3.7) the boundedness of

$$
\left\{\partial_{t} \rho_{\epsilon}\right\}_{\epsilon \in(0,1)} \text { in } L^{p^{\prime}}\left(0, T ; W^{-1} L_{\Phi_{\gamma}}(\Omega)\right), \gamma \geq 1 .
$$

Using the above bounded sequences we get (passing to subsequences if necessary)

$$
\begin{aligned}
\rho_{\epsilon} \stackrel{*}{\rightarrow} \rho \text { in } L^{\infty}\left(0, T ; L_{\Phi_{\gamma}}(\Omega)\right), \rho_{\epsilon} \rightarrow \rho \text { in } C\left([0, T] ; W^{-1} L_{\Phi_{\gamma}}(\Omega)\right), \\
D_{\epsilon} \widetilde{\mathbf{u}}_{\epsilon} \stackrel{\bar{M}}{\longrightarrow} \zeta, \mathbf{u}_{\epsilon} \rightarrow \mathbf{u} \text { in } L^{p}\left(0, T ; W^{1, p}(\Omega)^{3}\right) \cap L^{2}\left(0, T ; L^{2}(\partial \Omega)^{3}\right)
\end{aligned}
$$

for any $p \geq 1$, where

$$
\zeta=\left(\begin{array}{lll}
\partial_{1} u_{1} & \frac{\partial_{1} u_{2}+\partial_{2} u_{1}}{2} & \zeta_{13} \\
\frac{\partial_{1} u_{2}+\partial_{2} u_{1}}{2} & \partial_{2} u_{2} & \zeta_{23} \\
\zeta_{13} & \zeta_{23} & \zeta_{33}
\end{array}\right)
$$

In view of $(2.24),(3.10)$ and the condition $\left.\mathbf{u} \cdot \mathbf{n}\right|_{\partial \Omega \times(0, T)}=0$ we check $u_{3}=0$ and $\partial_{3} u_{1}=\partial_{3} u_{2}=0$ which imply $\mathbf{u}=\left(u_{1}, u_{2}, 0\right) \in L^{p}\left(0, T ; W^{1, p}(S)\right)$ for any $p \geq 1$. We can now see the reason for the choice of the function $h(\epsilon)$. If a limit of $\frac{h(\epsilon)}{\epsilon}$ does not exist then also the limit of the corresponding integral need not exist. If $\frac{h(\epsilon)}{\epsilon} \rightarrow \infty$ for $\epsilon \rightarrow 0$ then the limit function must be equal to zero on $(S \times\{0\}) \cup(S \times\{1\})$ and thus $\mathbf{u}=0$ which is not an interesting case. 
The convergence

$$
\rho_{\epsilon} \mathbf{u}_{\epsilon} \stackrel{\Psi_{\gamma}}{\longrightarrow} \rho \mathbf{u}, \gamma \geq 1
$$

immediately follows from $(3.9)_{2},(3.10)_{2}$ and from theorems about compact imbedding see $[\mathbf{1 6}]$.

Put now $\varphi(t) \psi(x)$, where $\varphi \in C_{0}^{\infty}(0, T)$ and $\psi=\left(\psi_{1}, \psi_{2}, 0\right) \in W^{1} L_{\Psi_{1 / 2}}(\Omega)^{3}$, $\left.\psi \cdot \mathbf{n}\right|_{\partial \Omega}=0$, as a test function in (2.23). Then

$$
\left|\int_{0}^{T} \varphi(t) \int_{\Omega} \rho_{\epsilon} \widetilde{\mathbf{u}}_{\epsilon} \otimes \widetilde{\mathbf{u}}_{\epsilon}: D_{\epsilon} \widetilde{\psi}_{\epsilon} d x d t\right| \leq
$$

$$
\left\|\rho_{\epsilon}\right\|_{L^{\infty}\left(0, T ; L_{\Phi_{\gamma}}(\Omega)\right)}\left\|\widetilde{\mathbf{u}}_{\epsilon}\right\|_{L^{p}\left(0, T ; L^{\infty}(\Omega)^{3}\right)}^{2}\|\psi\|_{1, \Psi_{\gamma}, \Omega}\|\varphi\|_{p^{\prime},(0, T)}, \quad \gamma \geq 1 .
$$

Since $w \in L_{\Phi_{1}}(\Omega \times(0, T))$ implies $w \in L_{\Phi_{1 / \alpha}}\left(0, T ; L_{\Phi_{(\alpha-1) / \alpha}}(\Omega)\right)$, which follows from the estimate

$$
\Phi_{1 / \alpha}\left(\Phi_{(\alpha-1) / \alpha}(z)\right) \leq 2 \Phi_{1}(z)+c, z \geq 0,
$$

and from the Jensen inequality, we arrive at

$$
\begin{gathered}
\left|\int_{0}^{T} \varphi(t) \int_{\Omega} P\left(\left|D_{\epsilon} \widetilde{\mathbf{u}}_{\epsilon}\right|\right) D_{\epsilon} \widetilde{\mathbf{u}}_{\epsilon}: D_{\epsilon} \widetilde{\psi}_{\epsilon} d x d t\right| \leq \\
c\|\psi\|_{1, \Psi_{1 / 2}, \Omega}\|\varphi\|_{\Psi_{1 / 2},(0, T)}\left(\int_{0}^{T} \int_{\Omega} \bar{M}\left(P\left(\left|D_{\epsilon} \widetilde{\mathbf{u}}_{\epsilon}\right|\right) D_{\epsilon} \widetilde{\mathbf{u}}_{\epsilon}\right) d x d t+1\right) .
\end{gathered}
$$

The estimates (3.12) and (3.13) represent "the worst integrals" in (2.23) thus we omit the estimates of the other ones. In view of the density of $C_{0}^{\infty}(0, T)$ in $E_{\Psi_{1 / 2}}(0, T)$ and the imbedding $L_{\Psi_{1 / \alpha}}(0, T) \hookrightarrow E_{\Psi_{1 / 2}}(0, T)$ for $\alpha>2$, we deduce from (2.23), (3.12) and (3.13) the boundedness of

$$
\left\{\partial_{t} \int_{0}^{1} \rho_{\epsilon} u_{i, \epsilon} d x_{3}\right\}_{\epsilon \in(0,1)} \text { in } L_{\Phi_{1 / \alpha}}\left(0, T ; W^{-1} L_{\Phi_{1 / 2}}(S)\right), i=1,2 .
$$

As a consequence of (3.11) and (3.14) (see also [16]) we find (passing to a subsequence if necessary)

$$
\int_{0}^{1} \rho_{\epsilon} u_{i, \epsilon} d x_{3} \rightarrow \int_{0}^{1} \rho u_{i} d x_{3} \text { in } C\left([0, T] ; W^{-1} L_{\Phi_{1}}(S)\right), i=1,2 .
$$

To treat the convective term we need the following proposition:

Proposition 2. Assume $\left\{\mathbf{u}_{\epsilon}\right\}_{\epsilon \in(0,1)}$ satisfies (3.4). Then (passing to a subsequence if necessary)

$$
\left\|u_{i, \epsilon}-\int_{0}^{1} u_{i, \epsilon} d x_{3}\right\|_{L^{p}\left(0, T ; L^{\infty}(\Omega)\right)} \rightarrow 0 \text { for } \epsilon \rightarrow 0 \text { and } i=1,2 .
$$

$\mathrm{P}$ r o o f: For if not then there exists a positive constant $c_{1}$ such that

$$
\left\|u_{i, \epsilon}-\int_{0}^{1} u_{i, \epsilon} d x_{3}\right\|_{L^{p}\left(0, T ; L^{\infty}(\Omega)\right)} \geq c_{1}
$$

for any $\epsilon$ sufficiently small. Hence it follows the existence of $I_{i, \epsilon, c_{1}} \subset(0, T)$ such that

$$
\left\|u_{i, \epsilon}(t)-\int_{0}^{1} u_{i, \epsilon}(t) d x_{3}\right\|_{\infty, \Omega} \geq \frac{c_{1}}{T^{1 / p}} \text { for a.a. } t \in I_{i, \epsilon, c_{1}} .
$$


In view of (3.4) we can also assume without loss of generality

$$
q^{1 / p}\left\|u_{i, \epsilon}(t)\right\|_{2, \partial \Omega}+\left\|D_{12} \mathbf{u}_{\epsilon}(t)\right\|_{p, \Omega} \leq c_{2} \text { for a.a. } t \in I_{i, \epsilon, c_{1}},
$$

where $c_{2}$ is sufficiently large and

$$
D_{12} \mathbf{u}_{\epsilon}:=\left(\begin{array}{ll}
\partial_{1} u_{1, \epsilon} & \frac{\partial_{1} u_{2, \epsilon}+\partial_{2} u_{1, \epsilon}}{2} \\
\frac{\partial_{1} u_{2, \epsilon}+\partial_{2} u_{1, \epsilon}}{2} & \partial_{2} u_{2, \epsilon}
\end{array}\right) .
$$

By contradictory arguments we can check that $\left|I_{i, \epsilon, c_{1}}\right| \geq c_{3}\left(i, c_{1}\right)>0$ for any small $\epsilon$. Under assumptions (3.18), (3.19) we can derive the special form of the Korn inequality

$$
\left\|u_{i, \epsilon}(t)-\int_{0}^{1} u_{i, \epsilon}(t) d x_{3}\right\|_{\infty, \Omega} \leq c\left(i, c_{1}, c_{2}\right)\left(\left\|D_{3} \mathbf{u}_{\epsilon}(t)\right\|_{p, \Omega}+\left\|u_{3, \epsilon}(t)\right\|_{2, \partial \Omega}\right)
$$

for any small $\epsilon$ and $t \in I_{i, \epsilon, c_{1}}$, where

$$
D_{3} \mathbf{u}_{\epsilon}=\left(\begin{array}{lll}
0 & 0 & \frac{\partial_{1} u_{3, \epsilon}+\partial_{3} u_{1, \epsilon}}{2} \\
0 & 0 & \frac{\partial_{2} u_{3, \epsilon}+\partial_{3} u_{2, \epsilon}}{2} \\
\frac{\partial_{1} u_{3, \epsilon}+\partial_{3} u_{1, \epsilon}}{2} & \frac{\partial_{2} u_{3, \epsilon}+\partial_{3} u_{2, \epsilon}}{2} & \partial_{3} u_{3, \epsilon}
\end{array}\right) .
$$

But as a consequence of (3.4) and Jegorov's theorem we get

$$
\left\|D_{3} \mathbf{u}_{\epsilon}(t)\right\|_{p, \Omega}+\left\|u_{3, \epsilon}(t)\right\|_{2, \partial \Omega} \rightarrow 0
$$

uniformly except for a small subset of $I_{i, \epsilon, c_{1}}$. (3.21) and (3.23) then contradicts (3.18).

Combining (3.10), (3.15) and (3.16) we conclude

$$
\rho_{\epsilon} u_{i, \epsilon} u_{j, \epsilon} \stackrel{\Psi_{\gamma}}{\longrightarrow} \rho u_{i} u_{j}, i, j=1,2, \gamma \geq 1 .
$$

Since the energy equality (2.26) and (2.17) imply

$$
P\left(\left|D_{\epsilon} \widetilde{\mathbf{u}}_{\epsilon}\right|\right) D_{\epsilon} \widetilde{\mathbf{u}}_{\epsilon} \stackrel{\Phi_{1}}{\overline{P(|\zeta|) \zeta},}
$$

we can perform the limit in $(2.22),(2.23)$ and (2.26) to derive

$$
\int_{0}^{T} \int_{S} \hat{\rho} \varphi_{t}+\hat{\rho} \mathbf{u} \cdot \nabla_{12} \varphi d x_{12} d t=0
$$

for any $\varphi \in \mathcal{D}\left(\mathbb{R}^{2} \times(0, T)\right)$, where $\nabla_{12}=\left(\partial_{1}, \partial_{2}\right), d x_{12}=d x_{1} d x_{2}, \hat{\rho}=\int_{0}^{1} \rho d x_{3}$ and $\mathbf{u}=\left(u_{1}, u_{2}, 0\right)$,

$$
\begin{gathered}
\int_{0}^{T} \int_{S}\left[\hat{\rho} \mathbf{u} \cdot \partial_{t} \psi+\hat{\rho} \mathbf{u} \otimes \mathbf{u}: D \psi+\hat{\rho} \operatorname{div}_{12} \psi\right] d x_{12} d t= \\
\int_{0}^{T} \int_{\Omega}[\overline{P(|\zeta|) \zeta}: D \psi-\rho \mathbf{f} \cdot \psi d x d t+ \\
q \int_{0}^{T} \int_{\partial S} \mathbf{u} \cdot \psi d S d t+2 h \int_{0}^{T} \int_{S} \mathbf{u} \cdot \psi d S d t
\end{gathered}
$$

for any $\psi=\left(\psi_{1}, \psi_{2}\right) \in C_{0}^{\infty}\left(0, T ; C^{\infty}(\bar{S})^{2}\right)$ such that $\left.\psi \cdot \mathbf{n}\right|_{\partial S \times(0, T)}=0$, where $\operatorname{div}_{12} \psi=\nabla_{12} \cdot \psi$ and $h=0$ if $h(\epsilon) \sim o(\epsilon)$ or $h$ is an positive constant if $h(\epsilon) \sim O(\epsilon)$, and

$$
\int_{S}\left[\frac{\hat{\rho}(t)|\mathbf{u}(t)|^{2}}{2}+\Phi_{1}(\hat{\rho})\right] d x_{12}+\int_{0}^{t} \int_{\Omega} P(|\zeta|)|\zeta|^{2} d x d s+q \int_{0}^{t} \int_{\partial S}|\mathbf{u}|^{2} d S d s
$$




$$
+2 h \int_{0}^{t} \int_{S}|\mathbf{u}|^{2} d S d s \leq \int_{0}^{t} \int_{\Omega} \rho \mathbf{f} \cdot \mathbf{u} d x d s+\int_{S}\left[\frac{\left|(\hat{\rho} \mathbf{u})_{0}\right|^{2}}{2 \rho_{0}}+\Phi_{1}\left(\rho_{0}\right)\right] d x_{12} .
$$

We now treat the nonlinearity in stress tensor. To derive the energy equality

$$
\int_{S}\left[\frac{\hat{\rho}|\mathbf{u}|^{2}}{2}+\Phi_{1}(\hat{\rho})\right] d x_{12}+\int_{0}^{t} \int_{\Omega} \overline{P(|\zeta|) \zeta}: D \mathbf{u} d x d s+q \int_{0}^{t} \int_{\partial S}|\mathbf{u}|^{2} d S d s=
$$

$$
\int_{0}^{t} \int_{\Omega} \rho \mathbf{f} \cdot \mathbf{u} d x d s+\int_{S}\left[\frac{\left|(\hat{\rho} \mathbf{u})_{0}\right|^{2}}{2 \rho_{0}}+\Phi_{1}\left(\rho_{0}\right)\right] d x_{12}
$$

we can use the same approach as in [11] based on the renormalized continuity equation (see [4]) and the Steklov function (see $[\mathbf{9}]$ ). In view of monotonicity of the function $P(|z|) z$ we get

$$
0 \leq \lim _{\epsilon \rightarrow 0} \int_{0}^{t} \int_{\Omega}\left(P\left(\left|D_{\epsilon} \widetilde{\mathbf{u}}_{\epsilon}\right|\right) D_{\epsilon} \widetilde{\mathbf{u}}_{\epsilon}-P(|T|) T\right):\left(D_{\epsilon} \widetilde{\mathbf{u}}_{\epsilon}-T\right) d x d s=
$$

$$
=\lim _{\epsilon \rightarrow 0} \int_{0}^{t} \int_{\Omega}\left(P\left(\left|D_{\epsilon} \widetilde{\mathbf{u}}_{\epsilon}\right|\right)\left|D_{\epsilon} \widetilde{\mathbf{u}}_{\epsilon}\right|^{2}-\overline{P(|\zeta|) \zeta}: T-P(|T|) T: \zeta+P(|T|)|T|^{2} d x d s,\right.
$$

for any symmetric tensor $T$. As a consequence of (2.26), (3.29), convexity and the Jensen inequality we deduce

$$
\begin{gathered}
\lim _{\epsilon \rightarrow 0} \int_{0}^{t} \int_{\Omega} P\left(\left|D_{\epsilon} \widetilde{\mathbf{u}}_{\epsilon}\right|\right)\left|D_{\epsilon} \widetilde{\mathbf{u}}_{\epsilon}\right|^{2} d x d s=\lim _{\epsilon \rightarrow 0}\left(-\int_{\Omega}\left[\frac{\rho_{\epsilon}(t)\left|\widetilde{\mathbf{u}}_{\epsilon}(t)\right|^{2}}{2}+\Phi_{1}\left(\rho_{\epsilon}\right)\right] d x-\right. \\
q \int_{0}^{t} \int_{\partial S \times(0,1)}\left|\widetilde{\mathbf{u}}_{\epsilon}\right|^{2} d S d s+\frac{h(\epsilon)}{\epsilon} \int_{0}^{t} \int_{(S \times\{0\}) \cup(S \times\{1\})}\left|\widetilde{\mathbf{u}}_{\epsilon}\right|^{2} d S d s \\
\left.\int_{0}^{t} \int_{\Omega} \rho_{\epsilon} \mathbf{f} \cdot \mathbf{u}_{\epsilon} d x d s+\int_{\Omega}\left[\frac{\left|\left(\rho_{\epsilon} \widetilde{\mathbf{u}}_{\epsilon}\right)_{0}\right|^{2}}{2 \rho_{0, \epsilon}}+\Phi_{1}\left(\rho_{0, \epsilon}\right) d x\right]\right)^{(2.27),(3.9)-(3.11),(3.24)} \leq \\
\quad-\int_{S}\left[\frac{\hat{\rho}(t)|\mathbf{u}(t)|^{2}}{2}+\Phi_{1}(\hat{\rho})\right] d x_{12}-q \int_{0}^{t} \int_{\partial S}|\mathbf{u}|^{2} d S d s-2 h \int_{0}^{t} \int_{S}|\mathbf{u}|^{2} d S d s+ \\
3.31) \int_{0}^{t} \int_{\Omega} \rho \mathbf{f} \cdot \mathbf{u} d x d s+\int_{S}\left[\frac{\left|(\hat{\rho} \mathbf{u})_{0}\right|^{2}}{2 \rho_{0}}+\Phi_{1}\left(\rho_{0}\right)\right] d x_{12} \stackrel{(3.29)}{=} \int_{0}^{t} \int_{\Omega} \frac{P(|\zeta|) \zeta}{P}: D \mathbf{u} d x d s .
\end{gathered}
$$

Hence and from (3.30) we conclude

$$
0 \leq \int_{0}^{t} \int_{\Omega}(\overline{P(|\zeta|) \zeta}-P(|T|) T)(D \mathbf{u}-T) d x d s
$$

and thus

$$
\int_{0}^{t} \int_{\Omega} \overline{P(|\zeta|) \zeta}: D \mathbf{v}=\int_{0}^{t} \int_{\Omega} P(|D \mathbf{u}|) D \mathbf{u}: D \mathbf{v} d x d s
$$

for any $\mathbf{v}=\left(v_{1}, v_{2}\right), \mathbf{v} \in L^{p}\left(0, T ; W^{1, p}(S)^{2}\right), D \mathbf{v} \in L_{M}(S \times(0, T))^{4}$ and $\mathbf{v}$. $\left.\mathbf{n}\right|_{\partial S \times(0, T)}=0$. The limit equations together with the energy equality thus read

$$
\int_{0}^{T} \int_{S} \hat{\rho} \varphi_{t}+\hat{\rho} \mathbf{u} \cdot \nabla_{12} \varphi d x_{12} d t=0
$$

for any $\varphi \in \mathcal{D}\left(\mathbb{R}^{2} \times(0, T)\right)$

$$
\int_{0}^{T} \int_{S}\left[\hat{\rho} \mathbf{u} \cdot \partial_{t} \psi+\hat{\rho} \mathbf{u} \otimes \mathbf{u}: D \psi+\hat{\rho} \operatorname{div}_{12} \psi\right] d x_{12} d t=\int_{0}^{T} \int_{S}[P(|D \mathbf{u}|) D \mathbf{u}: D \psi-
$$




$$
\rho \mathbf{f} \cdot \psi] d x d t+q \int_{0}^{T} \int_{\partial S} \mathbf{u} \cdot \psi d S d t+2 h \int_{0}^{T} \int_{S} \mathbf{u} \cdot \psi d S d t
$$

for any $\psi=\left(\psi_{1}, \psi_{2}\right) \in C_{0}^{\infty}\left(0, T ; C^{\infty}(\bar{S})^{2}\right)$ such that $\left.\psi \cdot \mathbf{n}\right|_{\partial S \times(0, T)}=0$ and

$$
\int_{S}\left[\frac{\hat{\rho}|\mathbf{u}|^{2}}{2}+\Phi_{1}(\hat{\rho})\right] d x_{12}+\int_{0}^{t} \int_{S} P(|D \mathbf{u}|)|D \mathbf{u}|^{2} d x d s+q \int_{0}^{t} \int_{\partial S}|\mathbf{u}|^{2} d S d s+
$$

$$
2 h \int_{0}^{t} \int_{S}|\mathbf{u}|^{2} d S d s=\int_{0}^{t} \int_{\Omega} \rho \mathbf{f} \cdot \mathbf{u} d x d s+\int_{S}\left[\frac{\left|(\hat{\rho} \mathbf{u})_{0}\right|^{2}}{2 \rho_{0}}+\Phi_{1}\left(\rho_{0}\right)\right] d x_{12} .
$$

From (2.16), (3.28), (3.31) and (3.33) it follows

$$
\begin{aligned}
& 0 \leq \int_{0}^{t} \int_{\Omega} P(|\zeta|)|\zeta|^{2} \leq \lim _{\epsilon \rightarrow 0} \int_{0}^{t} \int_{\Omega} P\left(\left|D_{\epsilon} \widetilde{\mathbf{u}}_{\epsilon}\right|\right)\left|D \widetilde{\mathbf{u}}_{\epsilon}\right|^{2} d x d s \\
& \leq \int_{0}^{t} \int_{S} P(|D \mathbf{u}|)|D \mathbf{u}|^{2} d x_{12} d s
\end{aligned}
$$

which together with the form of $\zeta$ (see (3.10) and bellow) give

$$
\int_{0}^{t} \int_{\Omega} P(|\zeta|)\left(\zeta_{13}^{2}+\zeta_{23}^{2}+\zeta_{33}^{2}\right) d x d s=0
$$

and thus $\zeta_{13}=\zeta_{23}=\zeta_{33}=0$.

It remains to prove $\rho=\rho\left(x_{1}, x_{2}\right)$. In view of (3.10) and the fact that $\zeta_{i 3}=0$, $i=1,2,3$, we can rewrite $(3.32)$ as

$$
0 \leq \int_{0}^{T} \int_{\Omega}(\overline{P(|\zeta|) \zeta}-P(|T|) T):(\zeta-T) d x d t
$$

for any symmetric tensor $T$. Put $T=\zeta-\lambda J$, where $J_{33}=\partial_{3} \varphi, \varphi \in \mathcal{D}(\Omega \times(0, T)$ and $J_{i j}=0$ otherwise. Then we arrive at

$$
\int_{0}^{T} \int_{\Omega}(\overline{P(|\zeta|) \zeta})_{33} \partial_{3} \varphi d x d s=\int_{0}^{T} \int_{\Omega} P(|\zeta|) \zeta_{33} \partial_{3} \varphi d x d s=0 .
$$

We further use $\psi=\left(0,0, \epsilon^{2} \psi_{3}\right), \psi_{3} \in \mathcal{D}(\Omega \times(0, T))$, as a test function in (2.23). It is clear that after the limit passage $\epsilon \rightarrow 0$ the integrals with stress tensors converge to zero because of (2.26), (3.25) and (3.39). We now only show that

$$
\rho_{\epsilon} u_{3, \epsilon} \frac{u_{3, \epsilon}}{\epsilon^{2}} \rightarrow 0 \text { in } \mathcal{D}^{\prime}(\Omega \times(0, T)),
$$

because it is the worst term in the remaining part of (2.23). As a consequence of the inequality

$$
\int_{0}^{T} \int_{\Omega} M\left(\frac{\left|u_{3, \epsilon}\right|}{\epsilon^{2}}\right) d x d t+\left\|\frac{u_{3, \epsilon}}{\epsilon^{2}}\right\|_{M, \Omega \times(0, T)} \leq c
$$

which follows from (2.26) and from zero traces of $u_{3, \epsilon}$, we can derive the estimate

$$
\begin{aligned}
& \left|\int_{0}^{T} \int_{\Omega} \rho_{\epsilon} u_{3, \epsilon} \frac{u_{3, \epsilon}}{\epsilon^{2}} \partial_{3} \psi_{3} d x d t\right| \leq c\left(\partial_{3} \psi_{3}\right)\left\|u_{3, \epsilon}\right\|_{M, \Omega \times(0, T)}\left\|\rho_{\epsilon} \frac{u_{3, \epsilon}}{\epsilon^{2}}\right\|_{\bar{M}, \Omega \times(0, T)} \leq \\
& c \epsilon^{2}\left(\int_{0}^{T} \int_{\Omega} \Phi_{1}\left(\rho_{\epsilon} \frac{\left|u_{3, \epsilon}\right|}{\epsilon^{2}}\right) d x d t+1\right) \leq c \epsilon^{2}\left(\int_{\left\{\rho_{\epsilon} \geq \frac{\left|u_{3, \epsilon}\right|}{\epsilon^{2}}\right\}} \Phi_{1}\left(\rho_{\epsilon} \frac{\left|u_{3, \epsilon}\right|}{\epsilon^{2}}\right) d x d t+\right.
\end{aligned}
$$




$$
\begin{gathered}
\left.\int_{\left\{\rho_{\epsilon}<\frac{\left|u_{3, \epsilon}\right|}{\epsilon^{2}}\right\}} \Phi_{1}\left(\rho_{\epsilon} \frac{\left|u_{3, \epsilon}\right|}{\epsilon^{2}}\right) d x d t+1\right) \leq c \epsilon^{2}\left(\int_{0}^{T} \int_{\Omega} \rho_{\epsilon} \frac{\left|u_{3, \epsilon}\right|}{\epsilon^{2}} \ln \left(1+\rho_{\epsilon}^{2}\right) d x d t+\right. \\
\int_{0}^{T} \int_{\Omega} \frac{\left|u_{3, \epsilon}\right|^{2}}{\epsilon^{2}} \ln \left(1+\frac{\left|u_{3, \epsilon}\right|}{\epsilon^{2}}\right) d x d t \leq c \epsilon^{2}\left(\int_{0}^{T} \int_{\Omega} M\left(\frac{\left|u_{3, \epsilon}\right|}{\epsilon^{2}}\right) d x d t+\right. \\
\left.\int_{0}^{T} \int_{\Omega} \bar{M}\left(\rho_{\epsilon} \ln \left(1+\rho_{\epsilon}^{2}\right)\right) d x d t+1\right) \leq c \epsilon^{2},
\end{gathered}
$$

because the estimate $\bar{M}\left(z \ln \left(1+z^{2}\right)\right) \leq c\left(\Phi_{2}(z)+1\right)$ holds for $z \geq 0$. After the limit passage $\epsilon \rightarrow 0$ in (2.23) we get

$$
\int_{0}^{t} \int_{\Omega} \rho \partial_{3} \psi_{3} d x d s=0
$$

\section{References}

[1] Aganovič I., Tutek Z.: A justification of the one-dimensional linear model of elastic beam, Math. Methods Appl. Sci. 8 (1986), No. 4, 502-515.

[2] Ciarlet P. G.: Mathematical Elasticity Volume II: Theory of plates, Studies in Mathematics and Its Applications 29, North-Holland Publishing Co., Amsterdam 2000

[3] Ciarlet P. G.: Mathematical Elasticity Volume III: Theory of shells, Studies in Mathematics and Its Applications 29, North-Holland Publishing Co., Amsterdam 2000.

[4] DiPerna R. J., Lions P. L.: Ordinary differential equations, transport theory and Sobolev spaces, Invent. Math., 98, 511-547 (1989)

[5] Feireisl E., Novotný A., Petzeltová H.: On the domain dependence of solutions to the compressible Navier-Stokes equations of a barotropic fluid, Math. Methods Appl. Sci. 25, No.12, 1045-1073 (2002)

[6] Iftimie D, Raugel G., Sell G. R.: Navier-Stokes equations in thin $3 D$ domains with Navier boundary conditions Indiana Univ. Math. J., 2007, 56(3) 1083-1156

[7] Jurak M., Tambača J.: Derivation and justification of a curved rod model, Math. Models and Methods Appl. Sci., 9 (1999), No. 7, pp. 991-1016

[8] Jurak M., Tambača J.: Linear curved rod model. General curve, Math. Models and Methods Appl. Sci., 11 (2001), No. 7, pp. 1237-1252

[9] Kufner A., John O., Fučík S.: Function spaces, Monographs and Textsbooks on Mechanics of Solids and Fluids. Mechanics: Analysis. Leyden: Noordhoff International Publishing. Prague: Publishing House of the Czechoslovak Academy of Sciences. XV, 454 p. (1977)

[10] Mamontov A. E.: Global solvability of the multidimensional Navier-Stokes equations of a compressible fluid with nonlinear viscosity I., Sib. Math. J. 40, No.2, 351-362 (1999)

[11] Mamontov A. E.: Global solvability of the multidimensional Navier-Stokes equations of a compressible nonlinear viscous fluid II., Sib. Math. J. 40, No.3, 541-555 (1999)

[12] Marušić S.: The asymptotic behaviour of quasi-Newtonian flow through a very thin or a very long curved pipe, Asymptotic Anal. 26, No.1, 73-89 (2001)

[13] Marušić S., Marušić-Paloka E.: Two-scale convergence for thin domains and its applications to some lower-dimensional models in fluid mechanics., Asymptotic Anal. 23, No.1, 23-57 (2000)

[14] Nazarov S. A.: Asymptotic solution of the Navier-Stokes problem on the flow of a thin layer of fluid Sib. Math. J.,1990, 31(2): 296-307

[15] Novotný A., Straškraba I.: Introduction to the mathematical theory of compressible flow, Oxford Lecture Series in Mathematics and its Applications 27. Oxford: Oxford University Press. xx, 506 p., (2004)

[16] Simon J.: Compact sets in the space $L^{p}(0, T ; B)$, Ann. Mat. Pura Appl., IV. Ser. 146, 65-96 (1987)

[17] Tiba D., Vodák R.: A general asymptotic model for Lipschitzian curved rods, Adv. in Math. Sc. and Appl. vol.15, No.1 (2005), pp.137-198

[18] Vodák R.: Asymptotic analysis of steady and nonsteady Navier-Stokes equations for barotropic compressible flow, submitted to Acta Applicandae Mathematicae

[19] Vodák R.: Justification of asymptotic two-dimensional models for steady Navier-Stokes equations for incompressible flow, submitted to Acta Applicandae Mathematicae 
Department of Mathematical Analysis and Applications of Mathematics, Faculty of Science, Palacky University, Tomkova 40, 77900 Olomouc-Hejčín, Czech Republic

E-mail address: vodak@inf.upol.cz 